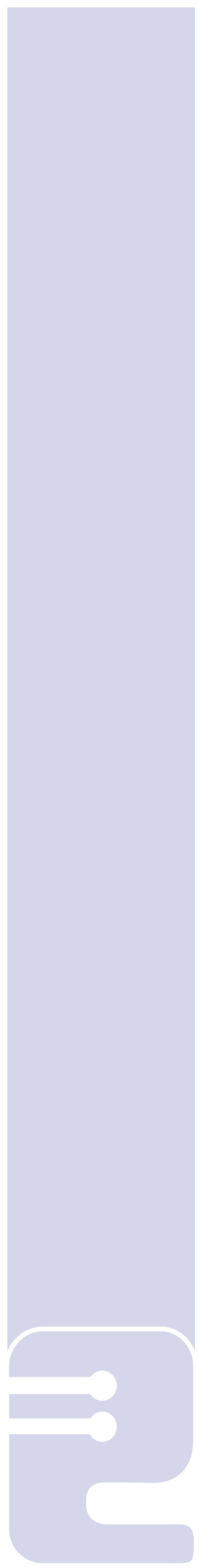

\title{
Insectos Asociados entre un Cultivo de Curuba y un Fragmento de Bosque Alto Andino de la Sabana de Bogotá
}

Daniela Espejo González; Jonhy Hidalgo Martin²; Maikol Santamaría Galindo³; Johanna Fernandez ${ }^{4}$

Recibido: Marzo 15 de 2014 Aprobado: Junio 16 de 2014

\section{Resumen:}

En el municipio de Madrid (Cundinamarca) se estableció un cultivo ecológico de curuba cerca de un Bosque Alto Andino y se realizaron muestreos semanales en bosque y cultivo para determinar los insectos asociados de importancia agroecológica. Se recuperaron 30 familias de insectos pertenecientes a los órdenes Diptera, Hymenoptera, Coleoptera, Hemiptera y Lepidoptera de las cuales 24 se hallaron en el bosque y 21 en el cultivo; 14 de ellas se consideraron de importancia agroecológica, debido a que están reportadas cumpliendo hábitos fitófagos, enemigos naturales o polinizadores. Del total de insectos recuperados considerados de importancia agroecológica el $30.99 \%$ correspondieron a fitófagos de las familias Tephritidae y Lonchaeidae (Diptera), Chrysomelidae y Curculionidae (Coleóptera), Cicadellidae (Hemiptera) y Nymphalidae (Lepidóptera). El 21.18\% correspondió a enemigos naturales de las familias Braconidae, Ichneumonidae, Diapriidae y Megaspilidae (Hymenoptera). El 47.83\% correspondió a polinizadores de las familias Halictidae y Apidae (Hymenoptera) y Tachinidae y Syrphidae (Díptera). El fragmento de bosque presentó mayor riqueza y abundancia de familias de insectos lo cual puede ser tenido en cuenta para procesos específicos de aprovechamiento sostenible de enemigos naturales y polinizadores en agroecosistemas.

Palabras clave: Biodiversidad, Passifloraceae, Servicios ecosistémicos, Polinizadores, Fitófagos.

\section{Abstract:}

In the municipality of Madrid (Cundinamarca) was established a curuba organically grown near a High Andean Forest and forest sampled weekly and culture to determine the insects associated agroecological importance. 30 families were recovered insects belonging to the orders Diptera, Hymenoptera, Coleoptera, Hemiptera and Lepidoptera of which 24 were found in the forest and 21 in the crop, 14 of them were considered agroecological importance because habits are reported meeting herbivores, pollinators or natural enemies. Of all the insects recovered considered important agroecological the $30.99 \%$ corresponded to phytophagous families Lonchaeidae and Tephritidae (Diptera), Chrysomelidae and Curculionidae (Coleoptera), Cicadellidae (Hemiptera) and Nymphalidae (Lepidoptera). The $21.18 \%$ were natural enemies of the families Braconidae, Ichneumonidae, Diapriidae and Megaspilidae (Hymenoptera). The $47.83 \%$ were pollinators of families Halictidae and Apidae (Hymenoptera) and Tachinidae and Syrphidae (Diptera). The forest fragment had higher richness and abundance of insect families which can be exploited for specific processes of sustainable use of natural enemies and pollinators in agroecosystems.

Keywords: Biodiversity, Passifloraceae, Ecosystem Services, Parasitoids, Pollinators, Phytophagous.

${ }^{1}$ Ingeniera en Agroecología. Corporación Universitaria Minuto de Dios. Grupo de Investigación Agroeco y Gestión Ambiental.

2Ingeniero en Agroecología. Corporación Universitaria Minuto de Dios. Grupo de Investigación Agroeco y Gestión Ambiental.

${ }^{3}$ Ingeniero en Agroecología. Magíster en Ciencias Agrarias. Corporación Universitaria Minuto de Dios. Grupo de Investigación Agroeco y Gestión Ambiental.

${ }^{4}$ Bióloga. Magíster en Ciencias Ambientales. Corporación Universitaria Minuto de Dios. Grupo de Investigación Agroeco y Gestión Ambiental. 


\section{INTRODUCCIÓN}

Las pasifloras ocupan uno de los renglones más importantes de la cadena frutícola (Conpes 2008; DANE 2011) y la curuba Passiflora tripartita var. mollissima (Nielsen \& Jorgensen) es una de las especies mas importantes, sembrada principalmente en los departamentos de Boyacá, Cundinamarca, Cauca y Nariño (MADR, 2014). Actualmente el manejo de la curuba en cultivo se ha basado en estrategias convencionales caracterizadas por el uso tipo calendario de insumos de síntesis química, que ha derivado en limitantes fitosanitarias principalmente por insectos fitófagos (plagas) (Coto \& Saunders 2004). El conocimiento de la biodiversidad de insectos representada en fitófagos, enemigos naturales y polinizadores es la base para el diseño de estrategias agroecológicas fundamentadas en el aprovechamiento de los servicios que ofrecen los ecosistemas naturales. Dentro de los servicios ecosistémicos se encuentran el control biológico y la polinización natural (Altieri \& Nicholls 2007). En el Altiplano Cundiboyacense los Bosques Altoandinos comprendidos entre 2900 y 3800 msnm proveen servicios ecosistémicos a las áreas de influencia, sin embargo, han sido fragmentados y desplazados por paisajes agropecuarios y plantaciones forestales de especies exóticas (Cortés et al. 1990; Rodríguez et al. 2004). Estos cambios en el uso de la tierra, pueden afectar las relaciones tróficas entre plantas e insectos. Por lo tanto, el objetivo de este estudio fue conocer la relación que hay entre un cultivo de curuba y un fragmento de Bosque Alto Andino, a través de la actividad de los insectos como fitófagos, enemigos naturales y polinizadores. Los resultados también permiten conocer la importancia de preservar un área de reserva forestal dentro de una finca productiva, que provee servicios ecosistémicos de regulación de las poblaciones de insectos.

\section{MATERIALES Y MÉTODOS}

\subsection{Localización}

La presente investigación se desarrolló en el Centro de Investigaciones y Transferencia de Biotecnología CITB Coraflor, el cual está ubicado en el municipio de Madrid (Cundinamarca), vereda Puente Piedra (Coordenadas: $4^{\circ} 49^{\prime} 43.02 " \mathrm{~N} ; 74^{\circ} 12^{\prime} 53.54^{\prime \prime}$ O) a una altitud de $2650 \mathrm{msnm}$.

\subsection{Caracteristicas del área de estudio}

Características del bosque. El bosque del CITB Coraflor cuenta con características propias de un Bosque Alto Andino típico de regiones con gradiente montañoso ubicadas entre los 2350 a 3500 msnm. (Rangel y Aguilar 1995), y que constituye una zona de ecotonía entre la vegetación cerrada de la media

\subsection{Material vegetal utilizado}

Características del cultivo de curuba. El área del cultivo fue de $1000 \mathrm{~m}^{2}$ con 55 plantas sembradas. El manejo del cultivo de curuba (nutrición, manejo de plagas y manejo cultural) estuvo sustentado por la normatividad vigente para la producción de alimentos ecológicos en Colombia, en concordancia con la Resolución 187 de 2006 del Ministerio de Agricultura y Desarrollo Rural.

\subsection{Muestreo de insectos}

El muestreo de insectos se orientó al conocimiento de fitófagos, enemigos naturales y polinizadores asociados al cultivo de curuba.

Selección de sitios de muestreo en el bosque y cultivo metodología de trampeo. Se realizó una selección y marcación de transeptos lineales, los cuales según Garaticeleya et al. (2012) son una tira de muestreo usada para el análisis en detalle de una determinada superficie, considerada representativa de una zona más amplia, esto para la ubicación de los diferentes tipos de trampas. En el bosque se trazaron 5 transeptos, cada uno a una distancia de $75 \mathrm{~m}$, intercalando la entrada por derecha o por izquierda con una desviación de 25 pasos. Se usaron trampas de caída, McPhail, red entomológica, malaise y luz (Figura 1). En el bosque fueron distribuidas una de cada una por cada transepto, excepto las trampas malaise y de luz las cuales se usaron una de cada una en la entrada del bosque. En el cultivo las trampas fueron distribuidas de forma homogénea en el área sembrada, excepto las trampas malaise y de luz que fueron instaladas una de cada una en el perímetro del cultivo.

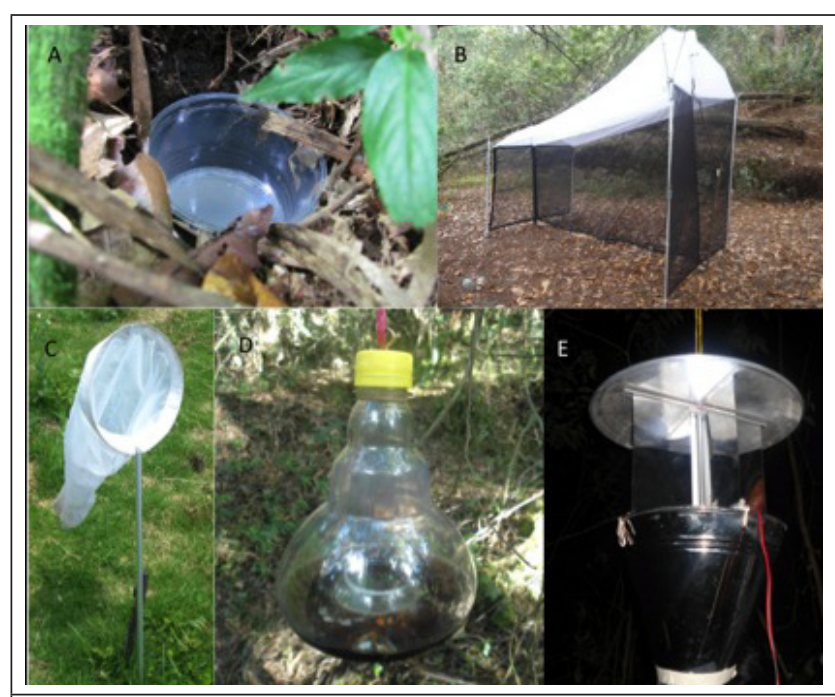

Figura 1. Tipos de trampas. A. Caida; B. Malaise; C. Red entomológica; D. McPhail; E. Luz. 
Almacenamiento y determinación taxonómica. Los insectos fueron almacenados en el Laboratorio de Ciencias de UNIMINUTO Bogotá, en recipientes rotulados con el tipo de trampa, fecha de colecta y número de transepto. La determinación taxonómica se realizó con base en caracteres taxonómicos hasta nivel de familia, y en algunos ejemplares hasta nivel de género. Indices de Margalef y Simpson fueron utilizados para determinar la riqueza y abundancia de familias.

\section{RESULTADOS Y DISCUSIÓN}

3.1 Insectos fitófagos de la curuba, sus enemigos naturales y polinizadores en el cultivo y el fragmento de bosque.

Insectos fitófagos: del orden Diptera se obtuvieron insectos de las familias Lonchaeidae y Tephritidae, los cuales son insectos de importancia económica en cultivos frutales en Colombia, que se alimentan de flores y frutos (Hernández 2003; Barranco 2003). Además se conocen como moscas de la fruta, las cuales son un limitante importante en cultivos de pasifloras, especialmente en curuba (Santamaría et al., 2014, ICA 2010; Umaña 2005; Castro et al., 2012) (Tabla 1). Del orden Coleoptera se obtuvieron insectos de las familias Chrysomelidae y Curculionidae, los cuales son fitófagos y defoliadores específicos asociados a diferentes familias de cultivos como Fabaceae, Malvaceae, Salicaceae, Passifloraceae y Verbenaceae (Arnett 1968; Anaya 1987; Costa et al. 1998). Del orden Hemiptera se obtuvieron insectos de la familia Cicadellidae, la cual se caracteriza por su aparato bucal chupador que ocasiona lesiones que producen manchas cloróticas y que evitan el desarrollo normal de las plantas jóvenes en cultivos de pasifloras (CORPOICA 2005; Salinas 2010) (Tabla 1). Del orden Lepidoptera se obtuvieron insectos de la familia Nymphalide, que en su estado de larva provoca una defoliación masiva, y elimina incluso las yemas laterales que impiden el crecimiento de las plantas (Salinas 2010) (Tabla 1). Las familias identificadas de los diferentes órdenes se presentaron en cultivo y bosque. Sin embargo Tephritidae solo se registró en el bosque, aunque especies de esta familia no son plaga clave en curuba. Cicadellidae y Nymphalidae solo se registraron en el cultivo. Lo anterior probablemente porque los Cicadellidae están asociados profundamente con pastos que son plantas que no proliferan de forma abundante en el bosque, y los Nymphalidae porque no encuentran los recursos alimenticios suficientes en el bosque, que si encuentran en el cultivo.

Insectos enemigos naturales: se determinaron enemigos naturales pertenecientes a los órdenes Hymenoptera y Diptera (Tabla 1). Del orden Hymenoptera se hallaron insectos de las familias Braconidae, Ichneumonidae, Megaspilidae y Diapriidae; las cuales se caracterizan por ser parasitoides, hiperparasitoides y depredadores que atacan principalmente dípteros, coleópteros y lepidíoteros (Matthews 1974; LaSalle \& Gauld 1991). En pasifloráceas avispas de las familias Braconidae y Diapriidae han sido registradas como importantes endoparasitoides de varias especies del género Dasiops (Lonchaeidae) (Quintero et al. 2012; Santamaría et al., 2012; Castro et al., 2012). Del orden Diptera se recuperaron insectos de la familia Syrphidae, de los cuales las larvas de al menos 14 géneros son depredadores importantes de varias plagas tales como áfidos, cóccidos, trips y larvas de mariposas (Vockeroth \& Thompson 1987; Núñez 1991; Nicholls 2008). El registro de enemigos naturales de plagas agrícolas asociadas a curuba en Bosque Alto Andino corrobora que la presencia de ecosistemas naturales es indispensable porque sirven de refugio para especies benéficas para la agricultura. Las familias determinadas como enemigos naturales fueron recuperadas tanto en el cultivo como en el bosque.

Insectos polinizadores: se determinaron insectos polinizadores pertenecientes a los órdenes Diptera e Hymenoptera (Tabla 1). Del orden Diptera se determinaron las familias Syrphidae y Tachinidae, las cuales son importantes ya que ocupan el primer y tercer lugar dentro de los dípteros polinizadores respectivamente (Gutierrez et al. 2005; Kevan y Baker 1983). Del orden Hymenoptera se determinaron las familias Apidae y Halictidae. Las abejas de la familia Apidae por su actividad polinizadora fomentan el mantenimiento de la diversidad vegetal y la producción de frutos y semillas (Fernández \& Sharkey 2006; APOLO 2010). La familia Halictidae, está compuesta en su mayoría por especies poliléticas, es decir que son himenópteros que visitan una amplia variedad de plantas para obtener néctar y polen (Engel 2000). Se registró la presencia de estas familias únicamente en el cultivo, sin embargo Bulla et al., (2013) observaron la visita de insectos de las familias Apidae y Halictidae a flores de pasifloras silvestres en Bosque Alto Andino. 


\begin{tabular}{|c|c|c|c|c|}
\hline \multicolumn{5}{|c|}{ Fitófagos } \\
\hline \multirow{2}{*}{ Orden } & \multirow{2}{*}{ Familia } & \multirow{2}{*}{ Hábito } & \multicolumn{2}{|c|}{ Hábitat } \\
\hline & & & Cultivo & Bosque \\
\hline \multirow[b]{2}{*}{ Diptera } & Tephritidae & $\begin{array}{l}\text { Adultos ovipositan en fruto. Larvas consumen } \\
\text { el interior del fruto }\end{array}$ & & $\mathrm{x}$ \\
\hline & Lonchaeidae & $\begin{array}{l}\text { Adultos ovipositan en botón floral y fruto. } \\
\text { Larvas consumen el interior del botón floral } \\
\text { y fruto }\end{array}$ & $\mathrm{x}$ & $\mathrm{x}$ \\
\hline Hemiptera & Cicadellidae & $\begin{array}{l}\text { Succiona savia y causa manchas cloróticas en } \\
\text { tejidos jóvenes }\end{array}$ & $x$ & \\
\hline Lepidoptera & Nymphalidae & Larvas consumen hojas & $x$ & \\
\hline \multirow{2}{*}{ Coleoptera } & Curculionidae & Adultos consumen hojas & $x$ & $\mathrm{x}$ \\
\hline & Chrysomelidae & Adultos consumen hojas & $x$ & $\mathrm{X}$ \\
\hline \multicolumn{5}{|c|}{ Enemigos Naturales } \\
\hline \multirow{2}{*}{ Orden } & \multirow{2}{*}{ Familia } & \multirow{2}{*}{ Hábito } & \multicolumn{2}{|c|}{ Hábitat } \\
\hline & & & Cultivo & Bosque \\
\hline \multirow{4}{*}{ Hymenoptera } & Braconidae & Ecto y endoparasitoide & $\mathrm{x}$ & $\mathrm{x}$ \\
\hline & Ichneumonidae & Ecto y endoparasitoide & $\mathrm{x}$ & $\mathrm{X}$ \\
\hline & Megaspilidae & Ecto y endoparasitoide & $x$ & $x$ \\
\hline & Diapriidae & Endoparasitoide y depredador & $\mathrm{X}$ & $\mathrm{X}$ \\
\hline Diptera & Syrphidae & Depredador & $x$ & $x$ \\
\hline \multicolumn{5}{|c|}{ Polinizadores } \\
\hline \multirow{2}{*}{ Orden } & \multirow{2}{*}{ Familia } & \multirow{2}{*}{ Hábito } & \multicolumn{2}{|c|}{ Hábitat } \\
\hline & & & Cultivo & Bosque \\
\hline \multirow{2}{*}{ Hymenoptera } & Apidae & Polinizador primario & $\mathrm{x}$ & \\
\hline & Halictidae & Polinizador secundario & $x$ & \\
\hline \multirow{2}{*}{ Diptera } & Syrphidae & Polinizador primario & $x$ & $\mathrm{x}$ \\
\hline & Tachinidae & Polinizador secundario & $\mathrm{x}$ & $\mathrm{x}$ \\
\hline
\end{tabular}

\subsection{Riqueza y abundancia}

Riqueza: está representada por el número de familias encontradas en los dos hábitats de estudio. El 78,01\% de los insectos encontrados correspondieron al orden Diptera, el $8,20 \%$ a Hymenoptera, el $7,07 \%$ al Hemiptera, el $4,57 \%$ a Coleoptera y el $2,16 \%$ al Lepidoptera (Tabla 2). Lo anterior concuerda con lo afirmado por Andrade y Amat (2000) quienes aseguran que el orden Diptera es ampliamente diverso en la Sabana de Bogotá. El orden Coleoptera tuvo el menor porcentaje, obedeciendo posiblemente a sus hábitos alimenticios que se ven perjudicados por los disturbios que ha sufrido el fragmento de bosque (inundaciones, talas, quemas, entre otros) (Sarasola et al. 2006). En el cultivo, la compactación del suelo y el riego constante también repercuten en la disminución de capturas de dicho orden; no cumpliendo con lo que aseveran los autores anteriormente mencionados. El orden Hymenoptera tuvo menor número de capturas que Diptera y Coleoptera probablemente debido a que según Smithers y Atkins (2001) la diversidad de éste orden disminuye en ambientes alto andinos. El valor de la riqueza calculado con el índice de Margalef para los 6 meses de muestreo fue de 6.35 para el fragmento de bosque y 5.92 para el cultivo, presentando mayor riqueza el fragmento de bosque ya que se reportó mayor número de familias en comparación con el cultivo. Esto se debe a que en el fragmento de bosque se encuentran las condiciones de equilibrio (hábitat para alimentación, reproducción y refugio) que albergan una mayor cantidad de especies de entomofauna (Nicholls 2008). Para el índice de Simpson, el fragmento de bosque presentó mayor valor de diversidad con 0.84 , mientras que el cultivo presentó índice de 0.76 . Lo anterior se debe a que el bosque es un ecosistema natural, poco intervenido en el cual existe mayor probabilidad de encontrar diversidad de insectos, sean o no de importancia agrícola.

Abundancia: de las 30 familias recuperadas, 14 familias se consideraron de importancia agroecológica 
para el caso de estudio, debido a que están reportadas cumpliendo diferentes hábitos como fitófagos, enemigos naturales, parasitoides 0 polinizadores (Tabla 3).

\begin{tabular}{|l|c|c|c|c|}
\hline \multicolumn{5}{|c|}{ Tabla 2. Número y porcentaje de insectos por orden en el cultivo y fragmento de bosque } \\
\hline \multicolumn{1}{|c|}{ Orden } & No. Individuos Cultivo & $\%$ & No. Individuos Bosque & $\%$ \\
\hline Diptera & 2838 & 72,92 & 4543 & 81,56 \\
\hline Coleoptera & 218 & 5,60 & 214 & 3,84 \\
\hline Lepidoptera & 48 & 1,23 & 156 & 2,80 \\
\hline Hymenoptera & 188 & 4,83 & 588 & 10,56 \\
\hline Hemiptera & 600 & 15,42 & 69 & 1,24 \\
\hline Total & 3892 & & 5570 & \\
\hline
\end{tabular}

Con relación a familias de importancia agroecológica, para el fragmento de Bosque Alto Andino las familias recuperadas en este estudio reportadas como fitófagos de la curuba presentan un $(21,28 \%)$, las familias de los enemigos naturales $(23,01 \%)$ y las familias que cumplen un rol ecológico polinizador (55.71\%); mientras que en el cultivo agroecológico de curuba las familias fitófagas presentan un $(63,44 \%)$, las de enemigos naturales $(15,05 \%)$ y las polinizadoras $(21,51 \%)$ (Tabla 3$)$. Lo anterior corrobora que el primer hábitat (bosque), se encuentra en equilibrio ecológico ya que en este existe una mayor diversidad vegetal en donde los vínculos tróficos entre poblaciones de artrópodos aumentan desarrollando sinergismos que promueven la estabilidad poblacional insectil (Southwood \& Way 1970), además los ecosistemas diversificados actúan como reservorio de fauna polinizadora debido a la disponibilidad de recursos ofrecidos (condiciones microclimáticas, agua, polen, sitios de reproducción, refugio, etc.). Por otro lado, el segundo hábitat (cultivo) presenta inestabilidad debido a que las poblaciones fitófagas cuentan con mayor número de especímenes respecto a los enemigos naturales; esto se debe a baja diversificación del sistema productivo, por ende la disponibilidad de recursos también se ve limitada (Altieri y Letourneau 1982, Flint y Roberts 1988).

\begin{tabular}{|c|c|c|c|c|c|}
\hline \multirow{2}{*}{ Rol ecológico } & \multirow{2}{*}{ Familia } & \multicolumn{2}{|c|}{ Bosque } & \multicolumn{2}{|c|}{ Cultivo } \\
\hline & & No. Individuos & $\%$ & No. Individuos & $\%$ \\
\hline \multirow{6}{*}{ Fitófagos } & Lonchaeidae & 387 & 15.57 & 58 & 7.80 \\
\hline & Tephritidae & 132 & 5.31 & 0 & 0.00 \\
\hline & Chysomelidae & 5 & 0.20 & 4 & 0.54 \\
\hline & Curculionidae & 5 & 0.20 & 9 & 1.21 \\
\hline & Cicadellidae & 0 & 0.00 & 384 & 51.61 \\
\hline & Nymphalidae & 0 & 0.00 & 17 & 2.28 \\
\hline Total & & 529 & 21.28 & 472 & 63.44 \\
\hline \multirow{4}{*}{ Enemigos naturales } & Braconidae & 411 & 16.53 & 47 & 6.32 \\
\hline & Ichneumonidae & 132 & 5.31 & 41 & 5.51 \\
\hline & Diapriidae & 26 & 1.05 & 5 & 0.67 \\
\hline & Megaspilidae & 3 & 0.12 & 19 & 2.55 \\
\hline Total & & 572 & 23.01 & 112 & 15.05 \\
\hline \multirow{4}{*}{ Polinizadores } & Halictidae & 0 & 0.00 & 15 & 2.02 \\
\hline & Apidae & 0 & 0.00 & 7 & 0.94 \\
\hline & Tachinidae & 1356 & 54.55 & 118 & 15.86 \\
\hline & Syrphidae & 29 & 1.17 & 20 & 2.69 \\
\hline Total & & 1385 & 55.71 & 160 & 21.51 \\
\hline \multicolumn{2}{|c|}{ Número total de individuos } & 2486 & 100.0 & 744 & 100.0 \\
\hline \multicolumn{2}{|c|}{ Número total de familias } & 10 & & 13 & \\
\hline
\end{tabular}

De acuerdo con Altieri \& Nicholls (2007), el manejo espacial de la vegetación y el mantenimiento de la vegetación no cultivada (fragmentos de bosque, corredores biológicos y arvenses) alrededor y dentro de los sistemas productivos, puede permitir que los agentes naturales de control biológico alcancen 
niveles de población más altos en huéspedes o presas alternativas y persistan en el medio agrícola; ya que éstos sirven como hábitats alternos para las poblaciones de enemigos naturales y polinizadores que van a repercutir en las áreas de cultivo en el mantenimiento de los niveles de plagas y el aumento en la tasa de polinización (Norris y Kogan 2005). Lo anterior se confirma con la diversidad encontrada de las familias de enemigos naturales y polinizadores pertenecientes a los órdenes Hymenoptera y Diptera.

\section{CONCLUSIONES}

Familias de insectos de importancia agroecológica como fitófagos (plagas), enemigos naturales y polinizadores están presentes tanto en el cultivo de curuba como en el fragmento de bosque alto andino, lo cual corrobora la importancia de los ecosistemas naturales en la sostenibilidad de los cultivos.

Se encuentra una importante diversidad y abundancia de insectos de las familias Inchneumonidae y Braconidae (Hymenoptera), los cuales son parasitoides de insectos de las familias Lonchaeidae y Tephritidae (Diptera) que se caracterizan por ser fitófagos limitantes de la producción de curuba.

El bosque es refugio de insectos polinizadores que probablemente se desplazan a los cultivos para polinizar flores de curuba y garantizar en un mayor porcentaje la fructificación del cultivo.

El uso sostenible de la biodiversidad es una de las alternativas más acertadas para la transformación de sistemas productivos convencionales a agroecológicos, ya que respeta los ciclos naturales de los ecosistemas y los aprovecha para el manejo de plagas; reduciendo al máximo el uso de insumos externos, como los agroquímicos.

\section{AGRADECIMIENTOS}

Esta publicación es producto del proyecto de investigación C112-38 "Determinación de insectos de importancia agroecológica para el cultivo de la curuba, asociados a un bosque alto andino de la Sabana de Bogotá" financiado por la Corporación Universitaria Minuto de Dios UNIMINUTO, a través de la Primera Convocatoria para el Desarrollo y Fortalecimiento de la Investigación en UNIMINUTO. Dr. Leonidas López Herrán, Rector General; Padre Harold Castilla, Rector Sede Principal. A la Dirección General de Investigaciones de UNIMINUTO, Dra. Amparo Vélez Ramírez, Directora General de Investigaciones. Se agradece el apoyo financiero, logístico y técnico de la Fundación de Asesorías para el Sector Rural Ciudad de Dios FUNDASES, Dr. Amilcar Salgado, Director de Fundases - CITB Coraflor y sus colaboradores. Se agradece a la Facultad de Ingeniería de UNIMINUTO, Dr. Manuel Dávila, Decano; Profesor Julio Rivera, Coordinador de Investigaciones. Al Programa de Ingeniería Agroecológica UNIMINUTO, Dr. Jhon Monje, Director; Dr. Álvaro Acevedo, Coordinador de Investigaciones IAGR. Al Instituto Colombiano Agropecuario C.I. Tibaitatá a través del Dr. Everth Ebratt Ravelo y Dra. Ángela Patricia Castro Ávila. A la Colección Taxonómica Nacional Luis María Murillo (C.I. Tibaitatá). A los Estudiantes investigadores de la Línea-Semillero en Protección Ecológica de Cultivos/ Red Ecológica.

\section{REFERENCIAS BIBLIOGRÁFICAS}

1. Altieri, MA; Letourneau, DK. 1982. Vegetation management and biological control in agroecosystems. Crop Protection 1: 405-430.

2. Altieri MA. \& Nicholls C. 2007. Diversidad y manejo de plagas en agroecosistemas, Pág. 34, 37.

3. Anaya, R. S. 1987. Crisomelinos del Valle de México. Tesis de Maestría, Colegio de Posgraduados Chapingo México 236 p.

4. Andrade, M. G. \& G. Amat. 2000. Guia preliminar de insectos de Santafé de Bogotá y sus alrededores. Departemento Técnico Administrativo Medio Ambiente. Alcaldia Mayor de Santafé de Bogotá.

5. Arnett, R. H. 1968. The beetles of the United States. A Manual for Identification. Ann. Arbor. Mich, The American Entomological Institute, U.S.A. 1112 pp.

6. Barranco, P. 2003. Dípteros de interés agronómico. Agromícidos plaga de cultivos hortícolas intensivos. Universidad de Almería.

7. Bulla J., Prieto J., Santamaría M., Fernández J., 2013. Insectos asociados a Passiflora longipes y Passiflora bogotensis en un fragmento de Bosque Alto Andino de la Sabana de Bogotá. Inventum. 15: 41-49.

8. Castro, A., E. Ebratt., y H. Brochero. 2012. Dasiops Rondani (Díptera: Lonchaeidae) asociadas a pasifloras cultivadas en Colombia. Tesis de Maestría en Ciencias Agrarias énfasis Entomología. Universidad Nacional de Colombia. Facultad de Agronomía. Bogotá. 
9. Coto, D; Saunders, JL. 2004. Insectos plagas de cultivos perennes con énfasis en frutales en América Central. Universidad Earth y el Centro Agronómico Tropical de Investigación y Enseñanza (CATIE).

10. CONPES, 2008. Política nacional fitosanitaria y de inocuidad para las cadenas de frutas y de otros vegetales. Ministerio de agricultura y desarrollo rural.

11. Corporación Colombiana de Investigación Agropecuaria (CORPOICA). 2005. Tecnología para el cultivo de la curuba. Manual técnico 6. Rionegro, Antioquia. Colombia.

12. Cortés, A., C. Chamorro \& A. vega, 1990. Cambio en el suelo por la implantación de praderas, coníferas y eucaliptos en un área aledaña al Embalse del Neusa (Páramo de Guerrero, Cundinamarca, Colombia.). IGAC, Investigaciones 2: $100-114$.

13. Costa, C.; S. A. Vanin e S. A. Casari-Chen. 1988. Larvas de Coleoptera do Brasil. Museu de Zoologia Universidade de São Paulo, São Paulo, Brasil.

14. Departamento Administrativo Nacional de Estadística -DANE. 2011. Resultados encuesta nacional agropecuaria ENA.

15. Engel, M. 2000. Classification of the bee tribe Augochlorini (Hymenoptera: Halictidae). Bulletin of the American Museum of Natural History 250: 1-1992.

16. Fernández, F. \& Sharkey, M (eds.). 2006. Introducción a los Hymenoptera de la Región Neotropical. Sociedad Colombiana de Entomología y Universidad Nacional de Colombia, Bogotá.

17. Flint, ML; Roberts, PA. 1988. Using crop diversity to manage pest problems: some California examples. American Journal of Alternative Agriculture 3: 164-167.

18. Garitaceleya Saldise, Natividad Gómez Corral, Martín López y Avilés Rodrigálvarez, 2012. Teoría y práctica del transecto como método de inventario para el sabinar (Juniperus thurifera).

19. Gutierrez, C. Carrejo, N. Ruíz, C. 2005. Listado de los géneros de Syrphidae (Diptera: Syrphoidea). Grupo de investigaciones entomológicas de la Universidad el Valle. Cali Colombia.

20. Hernández - Ortiz, v. 2003. Familia Tephritidae: Clasificación actual, relaciones filogenéticos y distribución de taxa americanos. En: XV Curso Internacional sobre moscas de la fruta. Memorias. Metapa de Domínguez, Chiapas, México. p.p 1123.
21. Instituto Colombiano Agropecuario (ICA), 2010. Plan nacional de detección, control y erradicación de moscas de la fruta (PNMF).

22. Kevan, P.G. \& Baker, H.G. 1983. Insects as flower visitors and pollinators. Annual Review of Entomology 28:407-445.

23. La Salle J., Gauld, I. 1991. Hymenoptera and Biodiversity crisis. Redia 74,315.

24. Matthews, R.W. 1974. Biology of Braconidae. Annual Review of Entomology 19: 15-32.

25. Ministerio de Agricultura y Desarrollo Rural MADR. 2014. Estadísticas Agropecuarias. Agronet. Recuperado de http://www.agronet.gov.co/agronet web1/estad\%C3\%ADsticas.aspx

26. Nicholls Clara, 2008. Control biológico de insectos: un enfoque agroecológico. Editorial Universidad de Antioquia.

27. Norris, RF. y Kogan, M. 2005. Ecology of interactions between weeds and arthropods. Annual review of entomology 50:479-503.

28. Núñez, E. 1991. Bases para el desarrollo del control integrado de los pulgones (Hom., Aphididae) de los cultivos de la provincia de León. Tesis doctoral. Universidad de León. Departamento de Biología Animal.

29. Observatorio de agentes polinizadores (APOLO), 2010. Polinizadores y biodiversidad. Asociación española de entomología, proyecto financiado por la Fundación biodiversidad, en su convocatoria 2010.

30. Quintero E. M., Lopez I. C., Kondo T., 2012. Manejo integrado de plagas como estrategia para el control de la mosca del botón floral del maracuyá Dasiops inedulis Steyskal (Diptera: Lonchaeidae), Manejo sanitario y epidemiología. Revista Corpoica - ciencia y tecnología agropecuaria.

31. Rangel,O. \& Aguilar, M. 1995. Una aproximación sobre la diversidad climática en las regiones naturales de Colombia. En: Rangel, O. (Ed.) Colombia, diversidad biótica l. Universidad Nacional de Colombia. Pág. 35.

32. Rangel, O. 2000. La región paramuna y franja aledaña en Colombia. En: Rangel, O. (Ed.) Colombia, diversidad biótica III. Universidad Nacional de Colombia.

33. Rodríguez, N., D. Armenteras, M.H.A. Rincón, M. Morales \& S. Sua. 2004. Forest biodiversity indicators in the Colombian Andes. Instituto de Investigación de Recursos Biológicos Alexander von Humboldt, Bogotá, Colombia. 
34. Salinas, H. 2010. Guía Técnica para el cultivo de "Maracuyá amarillo". Instituto de educación técnica profesional de roldanillo Valle.

35. Santamaría M., Ebratt E., Brochero H. 2012. Parasitoides naturales de moscas Dasiops (Diptera: Lonchaeidae) en pasifloras cultivadas de Colombia. Tesis de Maestría en Ciencias Agrarias énfasis Entomología. Universidad Nacional de Colombia. Facultad de Agronomía. Bogotá.

36. Santamaría M., Castro A., Ebratt E., Brochero H. 2014. Caracterización de daños de moscas del género Dasiops (Diptera: Lonchaeidae) en Passiflora spp. (Passifloraceae) cultivadas en Colombia. 67(1):7151-7162.

37. Sarasola Mauro M, Rusch Verónica E, Schlichter Tomás M, Ghersa Claudio M., 2006. Invasión de coníferas forestales en áreas de estepa y bosques de ciprés de la cordillera en la Región Andino Patagónica. Ecología Austral 16:143-156. Diciembre 2006 Asociación Argentina de Ecología.
38. Smithers. P. \& N. Atkins. 2001. Atitudinal variation in páramo invertebrate communities on volcan Chiles, with particular reference to carabidae (Coleoptera). In: Ramsay. P. M. (ed). The Ecology of Volcan Chilies: High-atitude ecosistems in de Ecuador Colombia border. Pebble \& Shell Publications.

39. Southwood, T. R. E. y M. J. Way. 1970. Ecological background to pest management. En Concepts of pest management. R. L. Rabb and F. E. Guthrie, eds. North Carolina State University, Raleigh, NC.

40. Umaña, M., 2005. Moscas de la fruta del género Dasiops (Diptera:Lonchaeidae) asociadas a la curuba y recomendaciones generales par su manejo agroecológico en la vereda Cañón, municipio de Sutamarchán - Boyacá. Revista colombiana de entomología.

41. Vockeroth J. R., F. C. Thompson. 1987. Syrphidae. Chapter 52 pp. 713-743 In: J. F. McAlpine, (ed.) Manual of the Nearctic Diptera, Vol. 2, Research Branch, Agriculture Canada monograph 28, Ottawa. 